\title{
Sentence recall with second stratum cues
}

\author{
RAYMOND W. KULHAVY, NANCY E. THORNTON, T. EMERSON HANCOCK, \\ and JAMES M. WEBB \\ Arizona State University, Tempe, Arizona
}

\begin{abstract}
As a test of the conjoint retention hypothesis, undergraduates heard 24 simple sentences while studying a map that represented 12 features as icons and labels, or as labels only. Control subjects saw a map outline without features. Half of the sentences were directly related to map features, and half were not related to features. Subject nouns were used as retrieval cues across three trials. Noun object recall of feature-related sentences was significantly better for all groups, but the predicted interaction between map and sentence type did not occur.
\end{abstract}

There is evidence that people can use spatial structures, such as reference maps, to facilitate the recall of related verbal information (see, e.g., Dean \& Kulhavy, 1981). The productive relationship between maps and associated verbal material has been explained in terms of the conjoint retention hypothesis (Kulhavy, Lee, \& Caterino, 1985). Conjoint retention is a version of dual coding theory, which assumes that maps are stored in memory as perceptual/spatial entities, and that verbal information is represented as a set of linguistic propositions primarily verbal in nature (Anderson, 1983). The dual coding approach also assumes that either representational store can be accessed from the other (Paivio, 1986).

Essentially, the conjoint retention hypothesis proposes that map features can be used as supplemental cues for retrieving related verbal information. In other words, when a map and related verbal stimuli are learned together, there is some probability that map elements can be used to access associated propositional knowledge. For example, when subjects receive a verbal test cue, they first attempt to locate the answer within the verbal/linguistic representational store. If this initial attempt fails, they have the option of using map information from the spatial store as a type of second-stratum cue for additional retrieval search. To the degree that such second-stratum cues are successful, there will be superior recall for verbal material directly related to map elements.

The conjoint retention notion leads to several predictions concerning spatial/verbal recall. First, because the spatial cues are specific to their associated propositions, we would expect verbal information directly related to map features to be better recalled than information that is not feature-related. Second, the more salient the representation of a map feature, the more likely it will be remembered and available for use as a retrieval cue. In the present study, we presented features either as labeled points, or as labeled points with mimetic icons

Correspondence may be addressed to Raymond W. Kulhavy, FPE0611, 322 Payne Hall, Arizona State University, Tempe, AZ 85287-0611. depicting the feature. Labels plus icons should produce superior recall compared to labels only, and both types of representation should outperform a control group seeing only the map boundaries.

\section{METHOD}

\section{Design and Subjects}

The form of map representation was varied between subjects to create three treatment conditions, with both test referent and trials treated as within-subjects variables. The base design was a 3 (map: icon-label vs. label only vs. boundary only) $\times 2$ (referent: feature-related vs. not feature-related) $\times 3$ (trials: 1 vs. 2 vs. 3) factorial, with repeated measures on the referent and trials factors.

The subjects were 60 undergraduate volunteers who participated for course credit. The subjects were randomly assigned, 20 to each betweensubjects condition, on the basis of the order in which they appeared for the experiment.

\section{Materials}

The icon-label map depicted a small Roman town of the classical period. The town was surrounded by a wall within which were three paths, one bisecting the area within the wall, and the others branching out from it toward the bottom and top of the town area. Spread about equally over the map domain were 12 features common to towns of this type. On the icon-lable version, each feature was marked by a mimetic drawing of the object, with the feature label printed directly adjacent to the drawing. Both the map and the feature icons were drawn by a professional artist. On the label-only map, the icons were removed and the labels remained in exactly the same location. The boundary-only map for the control group contained no icons or labels, and displayed only the wall and the three paths. The 12 labeled features, beginning with the top of the map and continuing to the right, were: barracks, plaza, cafe, capitol, steps, temple, markets, monument, arch, statue, fountain, and gate. All three versions of the map were printed on 21.50 $\times 27.95 \mathrm{~cm}$ heavy white paper for use in the experiment.

The verbal stimuli consisted of 24 simple, declarative sentences of the form noun-subject/verb/noun-object. All sentences contained exactly five words. In 12 of the sentences, the subject noun referred to one of the features depicted on the map (feature referents). In the remaining 12 sentences, the subject nouns were common landmarks that were not present on the map (no feature referents). The specific S-VO pairings were separately randomized four times to produce four different stimulus lists. Each of the four lists was randomly assigned to 5 subjects in each of the between-subjects conditions. The sentences were typed on individual index cards for use in the experiment.

Examples of sentences that are feature-referent and sentences that are not feature-referent, for one randomization, are:

1. The capitol protected the child. (referent)

2. The gate illustrated the period. (referent) 
1. The cemetery repelled the grocer. (nonreferent)

2. The villa covered the silver. (nonreferent)

\section{Procedure}

The subjects participated individually. They were randomly assigned to both map conditions and stimulus lists in the order of their appearance for the study. As the subjects reported for the experiment, they were assigned both a map from a randomly shuffled stack and a stimulus list (a card was drawn at random from a full deck; the suit determined the specific version of the list to be presented). When the subject was seated across a small table from the experimenter, the subject was told that he or she would be studying a map for $3 \mathrm{~min}$ as a prelude to learning a series of 24 sentences. The subjects were also informed that, following learning, they would be asked to recall the last part of the sentence when given the first part as a cue. Finally, the subjects were told that studying the map would help them learn the sentences.

During the learning phase, the subjects studied their version of the map while the experimenter read the 24 sentences at 10 -sec intervals. The order of sentences was randomized separately for each subject on each trial, and the time intervals were cued by audible clicks from a tape recorder. Following learning, there was a 30-sec rest period, after which the experimenter began the testing phase, which consisted of reading the noun subject for each sentence and recording the learner's verbatim response. The order in which the noun subjects were read was separately randomized for each experimental subject on each trial. The testing phase was not timed, and if a subject gave no response to a noun, he or she was prompted once with the question, "Do you remember anything about (noun subject)?" The respective map was on the table in front of each subject during the entire testing phase. The three trials followed one another immediately, and the procedures for each trial were identical.

\section{RESULTS AND DISCUSSION}

The sentence recalls were scored separately for recall of both noun object and noun object plus predicate. The results of both scorings were approximately the same, so only the noun object data will be reported here. The numbers of noun objects recalled correctly were entered into a 3 (map) $\times 2$ (referent) $\times 3$ (trials) analysis of variance, which yielded significance for the map $[F(2,57)=3.27$, $\left.M S_{\mathrm{e}}=17.75, p<.05\right]$, referent $\left[F(1,57)=54.38, M S_{\mathrm{e}}\right.$ $=4.60, p<.01]$, and trials $\left[F(2,114)=561.79, M S_{\mathrm{e}}\right.$ $=2.51, p<.01]$ main effects. The only interaction to reach conventional levels of significance was the feature

Table 1

Means and Standard Deviations for Correct Noun Object Recall, for Items Feature-Related and Items Not Feature-Related

\begin{tabular}{|c|c|c|c|c|c|c|}
\hline \multirow[b]{2}{*}{ Map Form } & \multicolumn{2}{|c|}{ Trial 1} & \multicolumn{2}{|c|}{ Trial 2} & \multicolumn{2}{|c|}{ Trial 3} \\
\hline & $M$ & $S D$ & $M$ & $S D$ & $M$ & $S D$ \\
\hline \multicolumn{7}{|c|}{ Feature } \\
\hline Icon-label & 5.30 & 1.34 & 10.21 & 1.32 & 11.35 & 1.04 \\
\hline Label only & 5.75 & 3.04 & 9.35 & 2.25 & 11.30 & 0.80 \\
\hline Boundary only & 3.70 & 2.05 & 8.10 & 2.63 & 10.21 & 1.50 \\
\hline \multicolumn{7}{|c|}{ Nonfeature } \\
\hline Icon-label & 2.90 & 1.71 & 7.75 & 3.16 & 10.05 & 2.28 \\
\hline Label only & 2.95 & 2.14 & 7.80 & 3.24 & 10.45 & 1.70 \\
\hline Boundary only & 2.10 & 2.08 & 6.65 & 3.35 & 9.62 & 2.28 \\
\hline
\end{tabular}

$\times$ trials effect $\left[F(2,114)=10.95, M S_{\mathrm{e}}=1.29\right.$, $p<.01]$. The means and standard deviations contributing to this analysis are reported in Table 1.

Post hoc Tukey HSD tests on the means from the three map conditions produced the following ranking: icon-label $=$ label only $>$ boundary only $(p<.05)$. The referent main effect resulted from higher performance on feature-related items, and recall increased significantly over trials. The referent $x$ trials interaction was due primarily to the more rapid acquisition of feature items over trials. Feature recall approached ceiling on Trial 2 and yielded only minor gains on the final measure, whereas performance on nonfeature items tended to increase steadily with each trial.

Presence of a map containing feature labels or labels plus icons increased the recall of sentence endings. The fact that such facilitation did not occur in the performance of the boundary-only condition provides partial support for the conjoint retention explanation. However, the hypothesized relationship between the map and referent factors did not materialize, although the form of the interaction was in the predicted direction. Hence, our data show a general positive influence for presence of feature maps, but provide no clear evidence of differential effects for sentence referents.

The overall higher recall of feature-related sentences was unexpected. It seems unlikely that such superiority occurred because feature-related items were intrinsically easier, since the predicate/object endings were assigned to noun subjects in four different randomizations. The remaining possibility is that this particular group of feature nouns were, in some fashion, more salient retrieval cues than their nonfeature counterparts.

The results of this experiment offer only weak support for the conjoint retention hypothesis. The general recall superiority for the feature map groups indicates that maps themselves have a positive effect on retrieval-a result that replicates previous research (e.g., Dean \& Kulhavy, 1981). However, the lack of differential effects between feature and nonfeature sentences as a function of map content does not substantiate the argument that specific features serve as additional cues for sentence retrieval.

\section{REFERENCES}

Anderson, J. R. (1983). The architecture of cognition. Cambridge, MA: Harvard University Press.

Dean, R. S., \& Kulhavy, R. W. (1981). The influence of spatial organization in prose learning. Journal of Educational Psychology, 73, 57-64.

Kulhavy, R. W., Lee, J. B., \& Caterino, L. C. (1985). Conjoint retention of maps and related discourse. Contemporary Educational Psychology, 10, 28-37.

PaIvio, A. (1986). Mental representations. New York: Oxford University Press.

(Manuscript received October 27, 1989.) 